\title{
A Mobile Device Controlled Colonoscope
}

\author{
Corey Sheerer ${ }^{1}$, Jung Hun Choi ${ }^{2, *}$ \\ ${ }^{1}$ Department of Mechanical Engineering, Ohio University, Athens, Ohio, U.S.A \\ ${ }^{2}$ Department of Mechanical Engineering, Georgia Southern University, Statesboro, U.S.A
}

Email address:

jchoi@georgiasouthern.edu (J. H. Choi)

${ }^{*}$ Corresponding author

\section{To cite this article:}

Corey Sheerer, Jung Hun Choi. A Mobile Device Controlled Colonoscope. International Journal of Mechanical Engineering and Applications. Vol. 8, No. 1, 2020, pp. 1-8. doi: 10.11648/j.ijmea.20200801.11

Received: October 14, 2019; Accepted: December 23, 2019; Published: January 8, 2020

\begin{abstract}
The background: Colorectal cancer is a prevalent and deadly disease that is further emerging within our society. As knowledge about this disease becomes more widespread, increasing demand for screening and high standards arises. This creates a need for a device which can be more easily mastered by physicians to achieve competency, as well as reduce injury risks from performing repetitive motions during a heavy load of colonoscopy procedures. Method: This research focuses on the fabrication of a prototype colonoscope that incorporates a holding structure to support the colonoscope and a hand-held device that actuates distal tip steering. Experimentation was performed to compare a learning curve of the prototype scope to a conventional scope. Results: Results show that subjects using the prototype scope did not experience any significant reduction in force in either the thumb or forearm. Cecal intubation time and learning rate showed no significant decrease while using the prototype scope compared to a conventional scope. Concluison: The prototype of the automated scope did not reduce learning rate or offset intubation time, it also did not increase the learning rate or, for the most part, increase intubation time compared to a conventional scope.
\end{abstract}

Keywords: Mobile Device, Colonoscopy, Intubation

\section{Introduction}

Colonoscopy is the primary method for detecting polyps and cancerous cells within the rectum and colon [1]. A colonoscope is inserted into the anus and travels up through the rectum and colon [2]. For a colonoscopy to be considered complete, the scope must reach the cecum (end of the large intestine). As the colonoscope advances and retracts through the colon, a physician examines the colon by means of video being transmitted through a camera attached to the tip of the scope [3]. If a polyp or other suspicious entity is observed, immediate removal can be performed [4].

Quality in colonoscopy can also be decreased when inexperienced physicians perform the colonoscopy before they reach competence. Competency for an endoscopist is most widely generalized as percentage, called the cecal intubation rate (CIR), of how often the physician reaches cecum with the colonoscope [5]. To be considered competent, an endoscopist must achieve a CIR of $90 \%$, although an experienced physician may achieve up to a $98 \%$ success rate $[3,5]$. Procedures that do not reach the cecum due to problems such as looping, intestinal fixations, or an inexperienced physician may leave potentially harmful areas unchecked for polyp formations [6].

While advancement in colonoscopy is occurring, it continues to be a complex procedure that involves extended training, experience, and focus in order to successfully and continually perform. Furthermore, with a growing awareness of the benefits screening has in the prevention and treatment of colorectal cancer, the magnitude of procedures being performed is rising [5]. This constitutes a need for a colonoscope design that can reduce learning time and improve the ergonomics of the scope in order to maintain high screening standards for an increasing number of procedures while protecting the physician's health.

Learning Rate of Colonoscopy

Learning colonoscopy incorporates many ethical and technical abilities to become competent as to provide a high standard of care. Learning colonoscopy is essential to perform complete colonoscopies within a reasonable time 
and entirely assess the colon. The American Board of Surgery requires a minimum of 50 procedures to be considered a competent endoscopist (most commonly defined as achieving a cecal intubation rate of $\geq 90 \%$ ), however, many studies show more training is required [7]. One study, by Church, J. et al, [5], concluded that after 18 students performed 125 procedures each, the cecal intubation rate was only $75.1 \%$ when the intubation time was limited to below 19 minutes. Another six-month study in Japan estimates a need for 325 to 350 procedures for residents to achieve competence: defined in the study as above a $90 \%$ cecal intubation rate with an insertion time of less than or equal to 15 minutes [8].

The most comprehensive study, Ward S. et al, [7], performed in the U.K., observed 297 trainees perform over 35,000 colonoscopies with database progress tracking. This study used two statistical strategies to assess cecal intubation rates: moving average method and learning curve cumulative summation (LC-CuSum). The moving average method calculated cecal intubation rates every 20 procedures performed by each resident. This resulted in an average of 233 procedures completed before a $90 \%$ cecal intubation rate was achieved. Progress of the residents can be seen in Figure 8. The LC-CuSum method observed competency in every procedure to determine if multiple predetermined competency thresholds were reached. As benchmarks were passed, parameters were adjusted in order to eliminate statistical bias arising from residents making mistakes earlier in their training. By the end of the study, only 36 of the 261 residents reached competency according to the LC-CuSum method. The residents who reached proficiency performed an average of 171 procedures to reach competency. This method was inconclusive because of the large number of residents who didn't complete proficiency, however, it indicates a much higher number of procedures are required to reach competence than is currently required. Many studies have been done to quantify the learning and competency rate achieved by residents. These studies indicate a relatively large number of procedures are required to become proficient at endoscopy and colonoscopy compared to the current standards. The American Board of Surgery's standard of 50 procedures needed to be considered competent is over 100 procedures less than any of the studies concluded procedure requirements to become competent. This shows a need for more training, better training methodology, and/or more intuitive medical device.

With the increasing awareness of the screening benefits to one's health as well as demand for colonoscopy procedures, expectations for physicians performing colonoscopy to adhere to high standards have risen. To meet these standards, a physician needs proper training and experience. Furthermore, the physician's health, in terms of colonoscopy injuries, cannot be compromised in order to maintain high standards in every procedure, as well as to perform a heavy load of colonoscopies.

There are two important factors for the colonoscopy and the aims of this research will compare the learning rate and intubation time between the conventional commercialized colonoscopes and the mobile device controlled colonoscope. The relation between two devices will be analyzed and quantified. Active colonoscopy training model will quantify the intubation time for both scopes and learning rate will be examed three times through fourteen subjects.

\section{Experimental Methods}

The colonoscope prototype aims to reduce physician fatigue and make steering the distal tip more intuitive $[9,10]$. Automated steering knobs controlled by a steering input mechanism have been included in the prototype to reduce fatigue of the left thumb and wrist, as well as be more intuitive. These inputs are for expansion of the device for if, and after, it has been verified as having high potential. The main components that will be added to the colonoscope are a hand-held steering device, a microcontroller, motors, and a stand. A high-level overview of the design showing the main components is seen in Figure 1. The complete design can be seen in Figure 2.

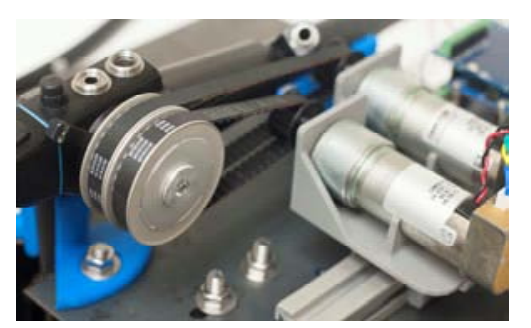

Figure 1. Belt system with two pulleys attached to the colonoscope shaft and held in place by a locknut piece.

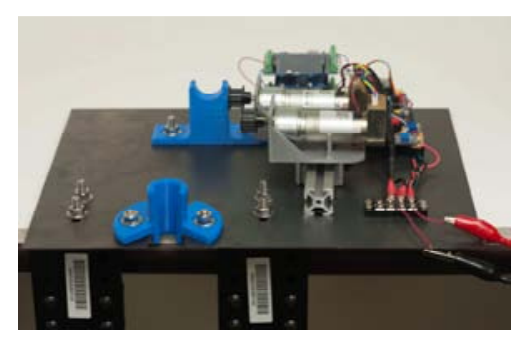

Figure 2. Prototype stand for colonoscope.

Four pieces are responsible for holding and stabilizing the colonoscope on the stand. The first piece (the cord support), seen in Figure 2, contains a slot running vertically down the side that is large enough to slip the universal cord of the colonoscope in and out of the part. The center of the cord support has a tapered hole running vertically through the part that sits flush with the top of the universal cord, which increases in diameter as it reaches the control head (upper cord). The intubation support holds the opposite side of the control head than the cord support, creating a simply supported system. The logic system of the prototype colonoscope controls the motors based off of user input. To ensure the steering knobs are not rotated past their limit, the system monitors rotation of each motor shaft and stops the motor as it reaches said limit. Furthermore, the system logic 
observes the torque being outputted by the motors and stops motor function if the torque becomes too high (meaning the distal tipoff the scope is pressing too hard against the colon wall).

The system contains two Arduino microcontrollers, a Bluetooth module, a motor shield, a current sensor, and two motors with encoders as seen in Figure 3. The first Arduino is responsible for receiving input through the Bluetooth module and executing said commands.

The second controller deals with monitoring and limiting the amount of rotation that can be achieved by the motors, as well as the amount of torque that is being outputted by the motors. Rotation is monitored using interrupt functionality on the Arduino to read encoders attached to each Pittman motors. Due to high precision encoders, the controller has a high amount of utilization, therefore, creating the need for the second controller to avoid lagging from when the user instigates a command to when it is performed. The complete architecture of the electro-mechanical interaction can be seen below in Figure 4.

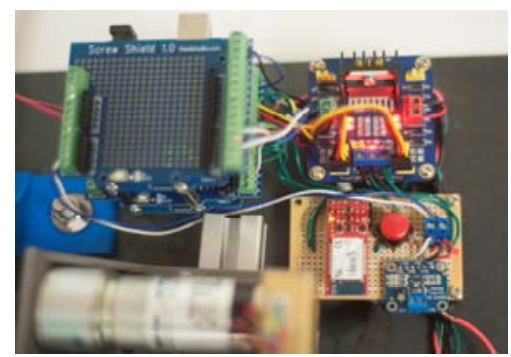

Figure 3. Circuit System with Arduino, motor shield, Bluetooth, current sensor, and motor with encoder.

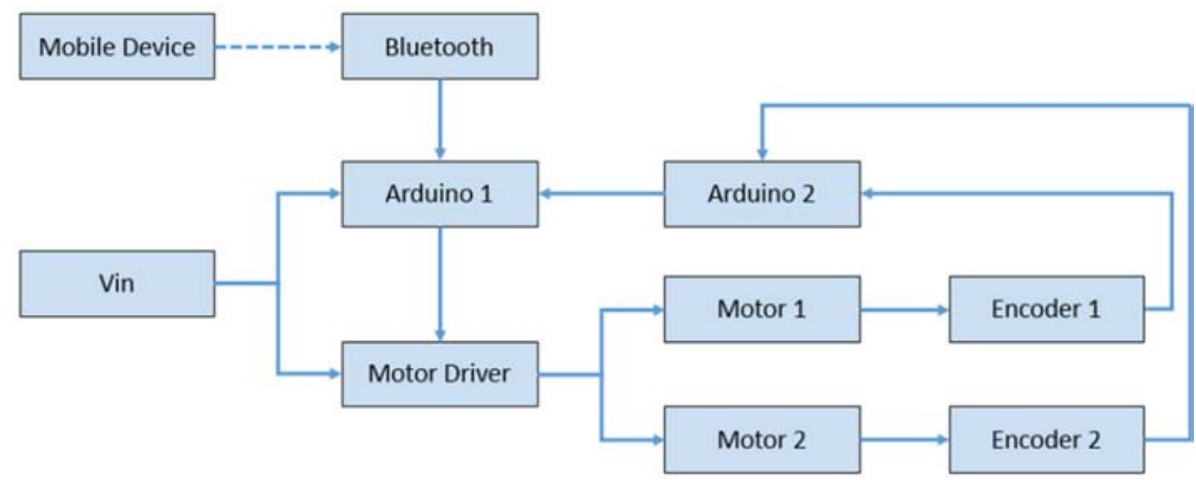

Figure 4. Electro-mechanical system block diagram.

It can be seen in Figure 4 that commands sent from the mobile device are received by the Bluetooth and passed to the first Arduino. Inputs that are received initiates control logic that is sent to the motor driver in order to perform the desired motor movement. Motor rotation is tracked by two encoders (one on each motor) connected to the second Arduino. If the steering knob limit is reached for either knob, defined by an encoder value reached, the second Arduino will send a signal to the first Arduino to prevent more motor rotation in that direction. The input steering device is an android phone with a custom application creating a client/server connection between the phone and the Bluetooth module connected to the Arduino. The mobile device hardware was chosen because of the ability to utilize all components needed to create a wireless steering system (i.e. user interface, input method, Bluetooth hardware), as well as pre-built libraries for programming.

\section{Learning Curve Evaluation}

The objective of the learning curve experimentation is provide data indicating that the prototype scope is more intuitive to control than a conventional scope. Intuitiveness was observed through two values: cecal intubation time and learning rate. These values were measured and compared between the two scopes as medical students performed multiple procedures on the Active Colonoscopy Training Model (a colon simulator) as seen in Figure 5 [11, 12]. Cecal intubation rate was considered as the duration from insertion of the scope into the simulated colon to reaching the end of the colon (cecum). Learning rate was classified as the amount of cecal intubation rate reduction between the first (baseline) and last cecal intubation time recorded [13].

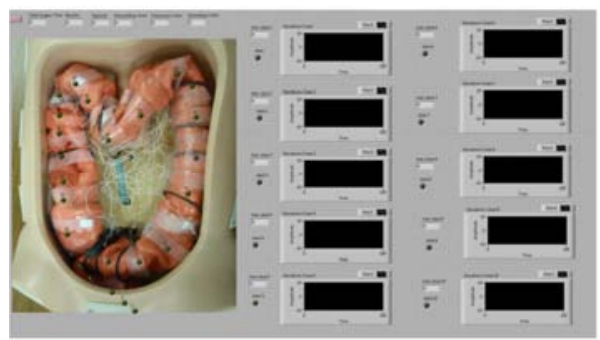

Figure 5. Active Colonoscopy Training Model simulator with LabView for force measurements [11].

\section{Recruitment and Procedure}

For this evaluation, 16 pre-medical students with no prior colonoscopy training or experience were recruited for this study. Recruitment consisted of voluntary participation from premedical students who receive a recruitment email including an overview about the study as well as the consent form attached. The students were split into two groups that determined which scope, the conventional or prototype scope, they used for learning and performing the experiment. Scope selection for each participant was assigned based on when the subject joined the study. A coin 
flip determined the scope that the first person used, and, thereafter, the scopes were alternately assigned to students as they joined the study. During their scheduled session, students performed four colonoscopy procedures on the colon simulator. When the participants arrived, they were given the consent form and allowed to ask any questions before beginning the test procedure. Before the testing started, participants were required to watch a training video containing information about how to use their specific scope to perform a colonoscopy, general information and guidelines about the procedure, as well as a demonstration with a professional endoscopist performing a colonoscopy on the simulator with their specific scope. After the video, the procedure for the colonoscopy trials proceeded as follows:

1. Participants were given two minutes to familiarize themselves with steering their scope.

2. Students started the colonoscopy procedure and a proctor began a stopwatch when the scope entered the simulator.

3. When the student reached the end of colon, time was recorded and the student was asked to remove the scope while keeping a clear view of the colon.

4. Steps 2 and 3 were repeated a total of four times.

Analysis

Two null hypotheses will be tested with the acquired data:

1. Using the prototype scope does not decrease the intubation time compared to a conventional scope.

2. Using the prototype scope does not increase learning rate of colonoscopy compared to the conventional scope.

A t-test with a $95 \%$ confidence was used to find significance between the mean intubation time means between the two scopes. Furthermore, normalized average intubation time between prototype and conventional scope on all four trials will be compared against each other to test the first null hypothesis. The intubation time over the four trials will be fit with a trend line, and a comparison between the slopes will be compared. This comparison will observe the differences in learning rate to test the second null hypothesis. Two plots will be included in this analysis: the first being a scatterplot showing the intubation over the four trials, and the second being a bar plot showing the last three intubation normalized with the first trial. The scatterplot will be fit with a best fit regression line in order to characterize the rate at which the students are improving their time. The second plot will contain $95 \%$ confidence intervals to visualize if the intubation time has significantly reduced from the initial (which will be referred to the baseline) procedure.

\section{Results}

Fourteen subjects performed the learning curve evaluation, however, two student were disqualified (one from each scope) after it was determined that they both produced multiple intubation time outliers over the four trials. The first subject that was disqualified, using the prototype scope, had high intubation times due to looping occurring within the colon model. The subject resulted in an outlier for both the second and third colonoscopy procedure trials. Furthermore, looping also affected the first trial, which did not result in an outlier, however, a noticeable increase in the intubation time for the sample data could be observed. Figure 6 displays a box-and-whisker plot showing the sample results for the four trials including the disqualified subject. The outliers can be seen on the second and third trials, with a large whisker on the first trial as a result from the disqualified subject. The second disqualification occurred on the conventional scope, where the third and fourth trial had a high intubation times that were both outliers. Similar to the prototype data, Figure 7 shows the distribution of the sample data over the four trials for the conventional scope.

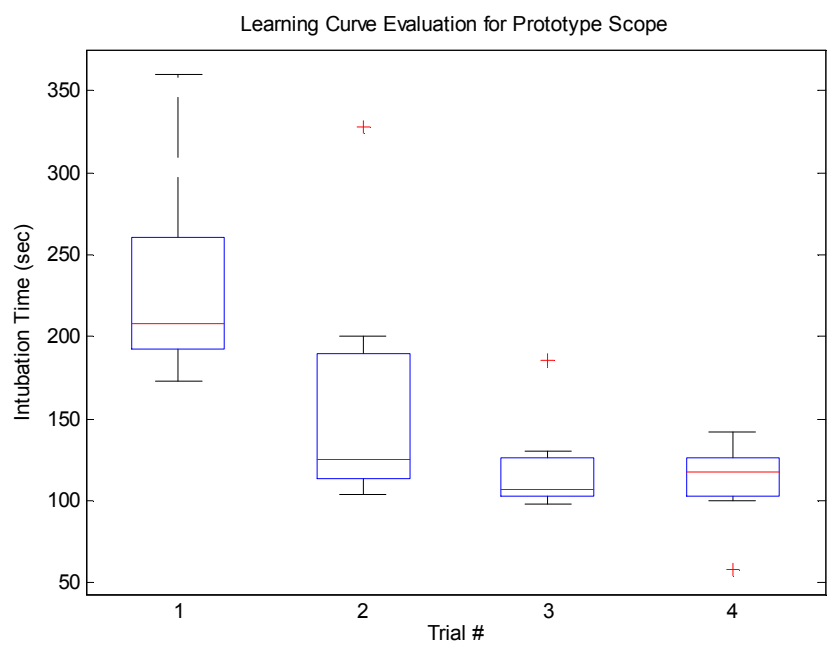

Figure 6. Prototype scope distribution of intubation time over the four trials.

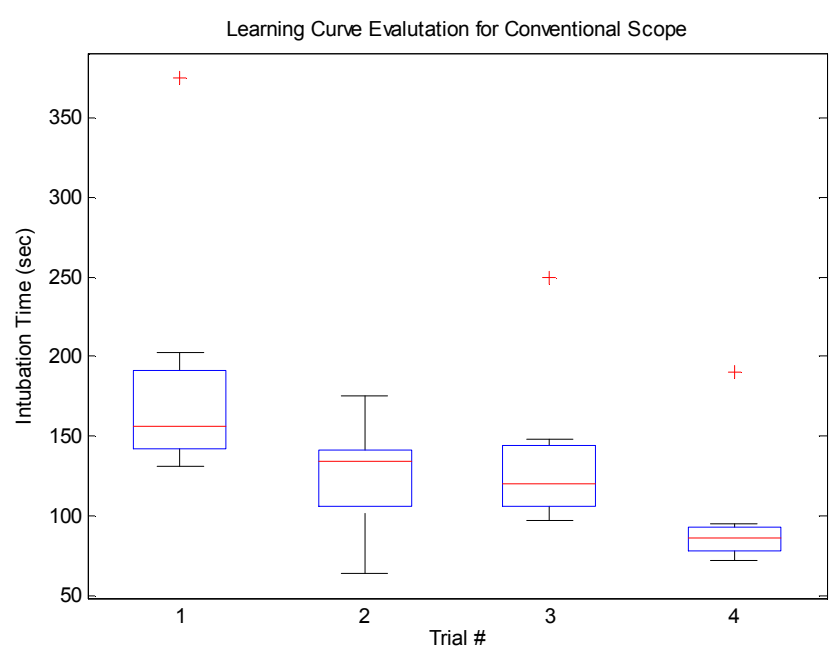

Figure 7. Conventional scope distribution of intubation times over the four trials.

Apart from the two outliers for both the prototype and conventional data-set caused by the disqualified subjects, a third outlier is present in both cases. These outliers are seen on the fourth trial of the prototype data-set (Figure 6), and the 
first trial of the conventional scope's data (Figure 7). Unlike the subjects that were disqualified, these subject had normal data for the other trials, and, in order to keep the number of subjects who performed the test on each scope equal to six, they were not disqualified themselves. Figure 8 shows the resulting average intubation times for the remaining six subjects on the conventional and prototype scope.

\section{Average Cecal Intubation Time}

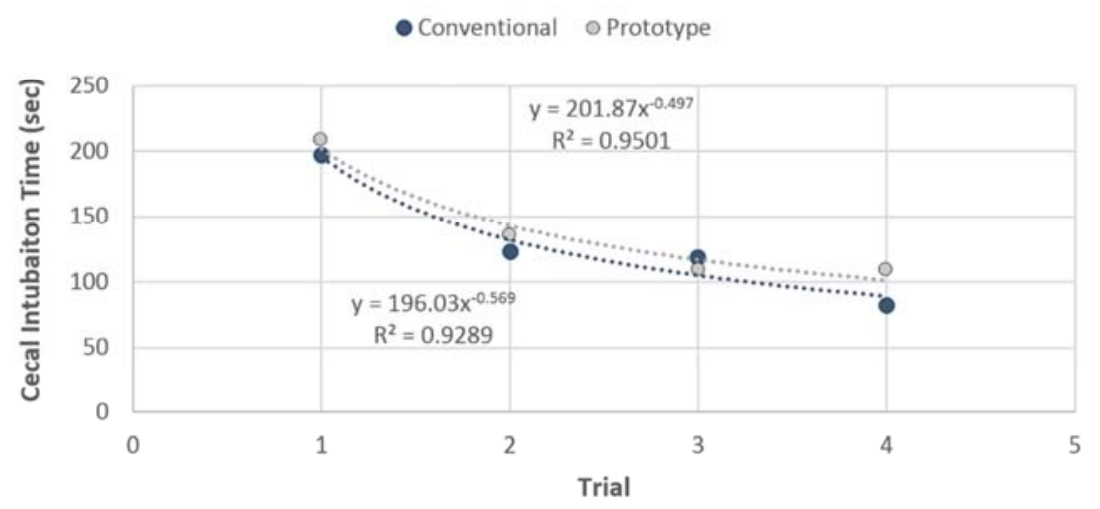

Figure 8. Average cecal intubation time for conventional and prototype scope.

Figure 8 shows similar intubation times for the conventional and prototype scope over the four trials. Fitting each curve with a power trend line in the form $\mathrm{Ax}^{-\mathrm{b}}$, the conventional and prototype data set resulted in a leading coefficient value of 196.03 and 201.87 respectively. Furthermore, the conventional and prototype case yielded a power of -0.569 and -0.497 respectively. The $R^{2}$ value associated with fit lines had values 0.929 and 0.950 for the conventional and prototype case respectively. Table 1 shows the average intubation times over the four trials for both scopes, as well as the t-test results between the means for each trial.

Table 1. Intubation time t-test results between conventional and prototype scope.

\begin{tabular}{lllll}
\hline Trial & $\mathbf{1}$ & $\mathbf{2}$ & $\mathbf{3}$ & $\mathbf{4}$ \\
\hline Prototype CIT & 209.67 & 136.33 & 109.5 & 109.5 \\
Conventional CIT & 197.5 & 123.33 & 119.67 & 83.17 \\
P-Value & 0.764 & 0.559 & 0.285 & 0.058 \\
\hline
\end{tabular}

Table 1 shows the first three trials showed no significant differences between the conventional and prototype scope with $p$-values of $0.76,0.56$, and 0.28 respectively. The fourth trial resulted in the average intubation time of 83.17 and 109.5 seconds for the prototype and conventional scope. This led to a p-value of 0.058 , bordering on significance. The normalized average intubation (normalized with the first trial) can be seen below in Figure 9.

Figure 9 shows the average cecal intubation time for the last three trials normalized with the first trial (baseline). It can be seen that both the prototype and conventional scope had large reductions in intubation time compared to the baseline procedure (a $52 \%$ intubation time reduction on the conventional scope and a $47 \%$ reduction on the prototype scope for the last trial). Furthermore, the 95\% confidence intervals on the graph show that every trial proceeding the first is significantly lower on both scopes. Table 2 shows the average normalized intubation time mean over the four trials for both scopes, as well as the t-test results between the means for each trial.

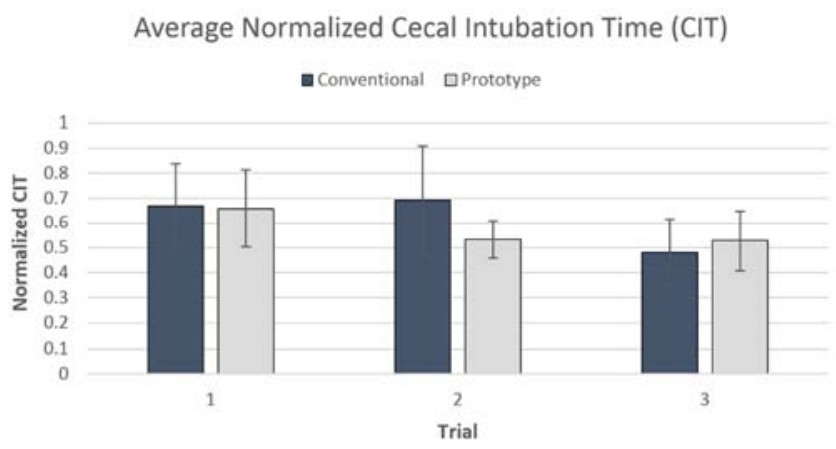

Figure 9. Average normalized cecal intubation time.

Table 2. Average Normalized intubation time t-test results.

\begin{tabular}{llll}
\hline Trial & $\mathbf{1}$ & $\mathbf{2}$ & $\mathbf{3}$ \\
\hline Prototype CIT & 0.659 & 0.533 & 0.528 \\
Conventional CIT & 0.668 & 0.693 & 0.481 \\
P-Value & 0.939 & 0.199 & 0.614 \\
\hline
\end{tabular}

It can be seen from Table 2 that none of the normalized intubation means for the trials comparing the two scopes were significantly different. The first and third trial had little difference between the normalized means (0.009 and 0.05 difference respectively). The second trial did have a larger difference, 0.16, compared to the other trials, however, also had the largest standard deviation for the conventional scope (0.245), reducing the significance between the means.

\section{Discussion}

A prototype colonoscope was fabricated and tested with regards to its ability to reduce fatigue and decrease learning rate of the colonoscopy procedure. One feature that worked well was the motor mount located on the linear slide in order to quickly remove the timing belts and remove the scope. 
This easily allowed the scope to be removed, which is important for disinfecting purposes after every procedure. Furthermore, it allows quick switching of scopes in between multiple procedures. Considering this, the locking mechanism for the slide could be improved (right now consisting of a nut that needs to be tightened with a wrench) to allow for locking of the slide with a simple lever, and eliminating the need for a tool.

The learning rate testing showed no significant difference between intubation time when comparing the prototype and conventional scope for any trials. This indicates that the prototype scope does not reduce intubation time or effect the learning rate of the colonoscopy procedure. The fourth trial did, however, resulted in p-value of 0.0584 , which borders on significance. This indicates that the average intubation time on the fourth trial may be different between the two scopes, and an increase in the amount of subjects would converge that $\mathrm{p}$-value into the significance range. With an average intubation time of 83.17 and 105.9 seconds on the last trial for the conventional and prototype scope respectively, and, considering these means to be significantly different, it can be concluded that the conventional scope allowed for faster times than the prototype scope once the subjects became more adept at controlling the scope.

Based on the trend lines fit onto the prototype and conventional scope, it can be seen that the conventional scope has a larger decreases in intubation time over the four trials. This difference may, again, be accounted for the offset of intubation time on the fourth trial between the two scopes. Removing the turning speed limitation on the prototype scope would possibly eliminate that offset, and, in turn, would make the difference in the decrease in intubation time over the four trials negligible between the two scopes. Considering the first three trials, as well as the argument stated above for the fourth trial, it can be concluded that the learning rate for both scopes is closely related.

While no significant difference occurred between the means of the intubation time, the normalized intubation times show both scopes had significant reductions in intubation times from the first (baseline) trial as seen by the $95 \%$ confidence interval not including the baseline time for either scope. Furthermore, no significance between the normalized data between the two scopes indicates that the reduction in intubation time with respect to the baseline value is not different between the scopes. This furthers the argument that the learning rate for both scopes is the same.

This conclusion is supported by Kuperij, et al, [14], who also performed a comparison on intubation time between a conventional and 'more intuitive' scope with modified grip and gesture steering of the distal tip. In this study, no significance was found between the two scopes' average intubation time on either of the two trials performed. However, Kuperij, et al, [14], did observe an offset between the means of the two scopes, where the designed scope had lower intubation times than the conventional in both trials, which was not seen in this test.

A second study, Xie, et al, [15], found similar results in regard to intubation time when comparing a variable stiffness colonoscope to a standard colonoscope: namely, there is no significance between the means. Cecal intubation rate (how often the cecum was reached), however, was significantly higher when using the variable stiffness scope. This indicates better versatility of the scope to help the physician overcome obstacles the colon may present in a procedure, including tight bends and obstructions. While intubation rate was not tested with prototype scope, it may have an advantage over the conventional scope in this regard due to its ability to navigate turns and obstacles without needing extra attention to control the scope as seen in the conventional scope. When a conventional scope needs to steer the distal tip, the force required to turn the steering knob will increase linearly corresponding the degree of the tip displacement. Furthermore, a force must be constantly applied to the steering knob in order to counteract the tension forces within the scope pulling the distal tip back to the neutral position. Considering these factors in controlling the two scopes, the prototype scope may be advantages in cecal intubation rate by reducing the effort and focus that is needed to be applied to moving the distal tip of the scope into a position that allows for advancement of the scope. This advantage would not have been observed in the learning rate assessment because of the relatively simple colon configuration used for testing.

A third study, Shanbhag, [16], had results contradicting the conclusions made from the previous studies. Shanbhag found that, while using a control head stand, intubation time was reduced by $32 \%$ compared to using a conventional scope without the stand [16]. The intubation time may have been reduced for this study because an improvement, which did not change anything fundamentally about steering the scope, was added to a design which has existed and proved its value over many years. In all the previous studies, a modification which drastically changes how the physician uses and controls the scope is added. This produces many new factors that play into learning and controlling the scope (all of which cannot be predicted when creating and/or designing the advancement). Shanbhag added no large underlying factors with a stand, which resulted in positive reduction in intubation times.

During testing, some factors may have impacted the intubation times, and therefore the test result, on either the prototype scope, the conventional scope, or both scopes. For instance, hand size and strength seemed to play a large role in how easily subjects could stabilize and control the conventional scope. Larger hand sizes could more easily reach the steering knobs and resulted in a more comfortable hand position. Subjects with smaller hands seemed to need and use more stabilization, which was achieved by resting the scope against their leg and/or table. Furthermore, subjects with smaller hands were required to stretch more with their thumb in order to reach the steering knobs. The prototype scope resolved the issues experienced on the conventional scope, however, created some factors of its own. 
The prototype scope, using the touchscreen to control the movement of the tip, created a need for subjects to look down at the device in order to find and press the buttons. The amount of times a subject looked down at the device varied greatly between subjects, as well as between the students performing the study an endoscopist who performed a few procedures with the prototype. The endoscopist seemed to have more of a need to look down and find the buttons compared to the students in the study. This difference may be caused by the age of the endoscopist compared to the younger students. Differences between the students could be hypothesized to be due to the type of phone the student currently uses, the amount of experience a student has using a touchscreen, and, perhaps, the experience the subject has with playing video games.

Personality and gender may also be a factor for intubation time on both scopes. For instance, males may have initially had more confidence in advancing the scope than females. Furthermore, personalities seemed to dictate the precision at which the subject centered the scope in the colon before advancing. Some subjects advanced the scope when they had a clear view of the lumen (but not necessarily centered), while others spent extra time completely centering the scope before advancing.

Considering all these factors, a reduction in intubation time may be achieved on the prototype scope compared to the conventional scope if more design was done on the prototype scope in order to eliminate or reduce said factors playing a role on the prototype scope. In this case, a design for a hand device for which users would not have to look down to locate the buttons would remedy the main problem for the prototype scope. However, this improvement would not guarantee that this will reduce the intubation time, as many factors such as personality and gender cannot be eliminated from the procedure.

\section{Conclusion}

While it can be concluded that the prototype scope did not reduce learning rate or offset intubation time, it also did not increase the learning rate or, for the most part, increase intubation time compared to a conventional scope. Furthermore, the scope provided a small device that was simple to hold (negligible stability needed) and could be easily and more uniformly manipulated by a variety of subjects compared to the conventional scope (i.e. hand size and strength had negligible impacts when controlling the prototype scope). Considering the experiment observed no decrease in learning rate or intubation time, it can be concluded that the proposed scope system has the ability to not to decrease efficiency in comparison to the conventional scope but effectively performed the intubation process. The prototype scope successfully allows a user to remotely control the mounted colonoscope, however, many improvements would be required to make a production model.

\section{References}

[1] M. A. Hayat, Colorectal Cancer, [Dordrecht]: Springer, 2009.

[2] Colonoscopy [electronic resource] / National Digestive Disease Information Clearinghouse, [Bethesda, Md]: U.S. Department of Health and Human Services, National Institutes of Health, National Institute of Diabetes, 2008.

[3] H. Messmann and J. Barnert, Atlas of Colonoscopy Examination Techniques and Diagnosis, Stuttgart; New York: Thieme, 2006.

[4] "Colon/Rectum Cancer | American Cancer Society," [Online]. Available:

http://www.cancer.org/cancer/colonandrectumcancer/. [Accessed 3 March 2014].

[5] J. Church, J. Oakley, J. Milsom, S. Strong and T. Hull, "Colonoscopy training: The need for patience (patients)," ANZ Journal of Surgery, vol. 72, no. 2, pp. 89-91, 2002.

[6] U. Hamdani, R. Naeem, F. Haider, P. Bansal, M. Komar, D. Diehl and H. Kirchner, "Risk Factors for Colonoscopic Perforation: A Population-Based Study of 80118 Cases," World Journal of Gastroenterology, vol. 19, no. 23, pp. 35963601,2013 .

[7] S. T. Ward, M. A. Mohammed, R. Walt, R. Valori, T. Ismail and P. Dunckley, "An Analysis of the Learning Curve to Achieve Competency at Colonoscopy Using JETS Database," Gut, 2014.

[8] K. Matsuda, Y. Yoshida, Y. Kawahara and H. Tajiri, "Tu1688 How Fast Does a Surgical Resident Learn Colonoscopy? Analysis of Competency in Colonoscopy for Ten Surgical Residents Supervised by Expert Endoscopists (Gastroenterologists) in the High-Volume Endoscopy Center in Japan," Gastrointestinal Endoscopy, vol. 75, no. 4, p. AB489, 2012.

[9] J. D. Litten, J. Choi and D. Drozek, "Development of a Colonoscopy Add-On Device for Improvement of the Intubation Process," Medical Devices, vol. 4, pp. 197-208, 2011.

[10] J. D. Litten, "Development of an Automatically Adjustable Colonoscope," Ohio University / OhioLINK, 2011.

[11] R. Kale, D. Koonce, D. Drozek and J. Choi, "Evaluation of Feedback Enabled Active Colonoscopy Training Model," Journal of Medical Devices, vol. 7, no. 4, pp. 041008-041008, 2013.

[12] R. Kale, J. Choi and D. Drozek, "Evaluation of Active Colonoscopy Training Model (ACTM)," Journal of Medical Devices, vol. 6, no. 1, pp. 017555-017555, 2012.

[13] S. Groth, D. K. Rex, T. Rosch and N. Hoepffner, "High Cecal Intubation Rates with a New Computer-Assisted Colonoscope: a Feasibility Study," The American Journal Of Gastroenterology, vol. 106, no. 6, pp. 1075-1080, 2011.

[14] N. Kuperij, R. Reilink, M. P. Schwartz, S. Stramigioli, Misra, Sarthak and I. A. M. J. Broeders, "Design of a User Interface for Intuitive Colonoscope Control," in IEEE, 2011.

[15] Q. Xie, B. Chen, L. Liu and H. Gan, "Does the VariableStiffness Colonoscope makes Colonoscopy Easier? A MetaAnalysis of the Efficacy of the Variable Stiffness Colonoscope Compared with the Standard Adult Colonoscope," BMC Gastroenterology, vol. 12, pp. 151-151, 2012. 
[16] R. Shanbhag, "Development of Medical Equipment to make Colonoscopy Procedures Safer for Physicians: Control Head Holder and Splatter Shield," Ohio University, 2014. 